\title{
Non-visual exploration of novel objects increases the levels of plasticity factors in the rat primary visual cortex
}

\author{
Catia M Pereira $^{1}$, Marco AM Freire ${ }^{2}$, José R Santos ${ }^{3}$, Joanilson S Guimarães ${ }^{4}$, Gabriella Dias-Florencio ${ }^{5}$, \\ Sharlene Santos ${ }^{5}$, Antonio Pereira ${ }^{6}$, Sidarta Ribeiro ${ }^{\text {Corresp. } 5}$ \\ 1 Instituto Internacional de Neurociências de Natal Edmond e Lily Safra, Macaiba, RN, Brasil \\ 2 Laboratório de Neurologia Experimental, Universidade Estadual do Rio Grande do Norte, Mossoró, RN, Brasil \\ 3 Departmento de Biociências, Universidade Federal de Sergipe, Itabaiana, SE, Brasil \\ 4 Instituto de Ciências Biológicas, Universidade Federal do Pará, Belém, PA, Brasil \\ 5 Instituto do Cérebro, Universidade Federal do Rio Grande do Norte, Natal, RN, Brasil \\ 6 Faculdade de Engenharia Elétrica, Universidade Federal do Pará, Belém, PA, Brasil \\ Corresponding Author: Sidarta Ribeiro \\ Email address: sidartaribeiro@neuro.ufrn.br
}

Background. Historically, the primary sensory areas of the cerebral cortex have been exclusively associated with the processing of a single sensory modality. Yet, the presence of tactile responses in the primary visual (V1) cortex has challenged this view, leading to the notion that primary sensory areas engage in cross-modal processing, and that the associated circuitry is modifiable by such activity. To explore this notion, here we assessed whether the exploration of novel objects in the dark induces the activation of plasticity markers in the V1 cortex of rats. Methods. Adult rats were allowed to freely explore for 20 minutes a completely dark box with four novel objects of different shapes and textures. Animals were euthanized either $1(n=5)$ or 3 hours $(n=5)$ after exploration. A control group $(n=5)$ was placed for 20 minutes in the same environment, but without the objects. Frontal sections of the brains were submitted to immunohistochemistry to measure protein levels of egr-1 and c-fos, and phosphorylated calcium-dependent kinase (pCaKMII), in V1 cortex. Results. The amount of neurons labeled with monoclonal antibodies against c-fos, egr-1 or pCaKMII increased significantly in V1 cortex after one hour of exploration in the dark. Three hours after exploration, the number of labeled neurons decreased to basal levels. Conclusions. Our results suggest that non-visual exploration induces the activation of immediate-early genes in V1 cortex, which is suggestive of cross-modal processing in this area. Besides, the increase in the number of neurons labeled with pCaKMII may signal a condition promoting synaptic plasticity. 


\section{Title:}

2 Non-visual exploration of novel objects increases the levels of plasticity factors in the rat

3 primary visual cortex

5 Running Title:

6 Non-visual exploration increases plasticity in V1 cortex

\section{Authors:}

9 Catia M Pereira' ${ }^{1,(5)}$, Marco Aurelio M Freire ${ }^{2,(5}$, Jose Ronaldo Santos ${ }^{3}$, Joanilson S Guimarães ${ }^{4}$,

10 Gabriella Dias-Florencio ${ }^{1}$, Sharlenne Santos $^{1}$, Antonio Pereira ${ }^{5}$, Sidarta Ribeiro ${ }^{6, *}$

\section{Affiliations:}

$13{ }^{1}$ Edmond Lily Safra International Institute of Neuroscience of Natal (ELS-IINN), Natal/RN 14 Brazil

$15{ }^{2}$ Postgraduation Program in Health and Society, University of the State of Rio Grande do Norte 16 (UERN), Mossoró/RN - Brazil

$17{ }^{3}$ Laboratory of Behavioral and Evolutionary Neurobiology, Department of Biosciences, Federal 18 University of Sergipe (UFS), Itabaiana/SE - Brazil

$19{ }^{4}$ Laboratory of Experimental Neuroprotection and Neuroregeneration, Institute of Biological 20 Sciences, Federal University of Pará (UFPA), Belém/PA - Brazil

$21{ }^{5}$ Faculty of Electrical Engineering, Institute of Technology, Federal University of Pará (UFPA), 22 Belém/PA - Brazil

$23{ }^{6}$ Brain Institute, Federal University of Rio Grande do Norte (UFRN), Natal/RN - Brazil

24 (5) These authors contributed equally to this work

25

$26{ }^{*}$ Corresponding author

27 Sidarta Ribeiro, PhD.

28 Brain Institute, Federal University of Rio Grande do Norte (UFRN), Av. Nascimento de Castro 29 2155, Natal/RN - Brazil

30 Email address: sidartaribeiro@neuro.ufrn.br 


\section{Abstract}

34 Background. Historically, the primary sensory areas of the cerebral cortex have been exclusively associated with the processing of a single sensory modality. Yet, the presence of tactile responses in the primary visual (V1) cortex has challenged this view, leading to the notion that primary sensory areas engage in cross-modal processing, and that the associated circuitry is modifiable by such activity. To explore this notion, here we assessed whether the exploration of novel objects in the dark induces the activation of plasticity markers in the V1 cortex of rats.

40 Methods. Adult rats were allowed to freely explore for 20 minutes a completely dark box with 41 four novel objects of different shapes and textures. Animals were euthanized either $1(n=5)$ or 3 42 hours $(n=5)$ after exploration. A control group $(n=5)$ was placed for 20 minutes in the same 43 environment, but without the objects. Frontal sections of the brains were submitted to 44 immunohistochemistry to measure protein levels of egr-1 and c-fos, and phosphorylated calcium45 dependent kinase (pCaKMII), in V1 cortex.

46 Results. The amount of neurons labeled with monoclonal antibodies against c-fos, egr-1 or 47 pCaKMII increased significantly in V1 cortex after one hour of exploration in the dark. Three 48 hours after exploration, the number of labeled neurons decreased to basal levels.

49 Conclusions. Our results suggest that non-visual exploration induces the activation of 50 immediate-early genes in V1 cortex, which is suggestive of cross-modal processing in this area. 51 Besides, the increase in the number of neurons labeled with pCaKMII may signal a condition 52 promoting synaptic plasticity. 


\section{Introduction}

55

56

57

58

At the beginning of the last century, Korbinian Brodmann proposed that the neocortex is divided into several cytoarchitectonically distinct areas, each one devoted to a distinct function (Brodmann, 2006; Guimaraes et al., 2016). This classical finding was subsequently reinforced by several studies using multiple experimental techniques, including tract-tracing studies showing that thalamocortical inputs from a given sensory modality preferentially target specific primary cortical areas (Hubel \& Wiesel, 1959; Mountcastle, 1997; Pereira et al. 2000; Rocha et al., 2007; Santiago et al., 2007; Dooley et al., 2015). However, a growing number of recent studies have shown that neuronal responses in those areas are characterized by a large amount of sensory crosstalk (Lemus et al., 2010; Vincis \& Fontanini, 2016). For instance, whisker stimulation in rats generates patterns of subthreshold cortical activation that spread far from the traditional limits of the primary somatosensory cortex (Frostig et al., 2008). This pattern of activation means that unimodal sensory stimulation can potentially modulate the activity of neurons located in sensory areas related to other sensory modalities, despite the existence of cytoarchitectural borders (Stehberg et al., 2014).

It remains to be determined, however, whether such cross-modal interactions would impact beyond the transitory modulation of neuronal activity, to trigger longer-lasting plastic changes in primary sensory cortices. Monocular enucleation during adulthood elicits cross-modal reorganization of the visual cortex, a phenomenon supported by preexisting projections from the somatosensory cortex (Van Brussel et al., 2011). Thus, cross-modal plasticity may help individuals recover from sensory deprivation due to injury of the neural pathways associated with a specific modality (Rabinowitch \& Bai, 2016).

In a previous study, we showed that the whisker-based exploration of novel objects in the darkness leads to a marked upregulation of the mRNA levels of calcium-dependent immediateearly genes (IEGs) in primary visual (V1) cortex of calcium-dependent immediate-early genes (IEGs) which are associated with memory consolidation (Ribeiro et al., 2007). Using multielectrode recordings and the same free-exploration paradigm, we also showed in a later study that $35 \%$ of the V1 neurons significantly change their firing rates during whisker-based object exploration, despite the lack of visual inputs (Vasconcelos et al., 2011). Most importantly, neuronal ensembles in V1 cortex were shown to carry non-visual information useful to discriminate among different objects (Vasconcelos et al., 2011). 
85

86

87

88

89

90

91

92

93

94

95

96

97

98

99

100

101

102

103

104

105

106

107

108

109

110

111

112

113

114

As a follow up to these earlier studies, in the present work we sought to contribute with additional information concerning the modulation of visual cortex induced by tactile stimulation. We investigated the causes and consequences of cross-modal mRNA IEG induction in V1 cortex, by assessing the levels of phosphorylated CaMKII, a calcium-dependent kinase upstream of $c$-fos and egr-l transcripts with a well-documented role in plasticity (Kaczmarek \& Chaudhuri, 1997). To examine the response downstream, we assessed the levels of c-fos and egr1 proteins (Bading et al., 1993; Kaczmarek, 2000; Jones et al., 2001; Nunes et al., 2010).

\section{Materials and Methods}

Animals: Fifteen adult male Wistar rats (5 months-old, $320 \pm 20 \mathrm{~g}$ ) were used in this research. All experimental procedures were approved by the Institutional Local Ethics Committee for the Use of Animals (ID 05/2007), in accordance with the NIH Guidelines for the Care and Use of Laboratory Animals. All efforts were made to avoid suffering and to reduce the number of animals used. Prior to the experiment, all animals remained in conventional cages $(49 \times 34 \times 16$ $\mathrm{cm}$ ) under standard conditions (lights on at 07:00, lights off at 19:00, $22 \pm 2^{\circ} \mathrm{C}$ ) with free access to food and water.

Object exploration in the dark: All experimental procedures began at 22:00 under infrared light, to ensure that the animals were kept unstimulated by visible light for $3 \mathrm{~h}$. A group of animals $(n=10)$ was transferred to a closed box $(55 \times 40 \times 20 \mathrm{~cm})$ and allowed to freely explore four novel objects of different shapes and textures (termed 'ball', 'urchin', 'brush' and 'food') for $20 \mathrm{~min}$ (Fig. 1A). The animals were then returned to their home cages and euthanized after one $(n=5)$ or three $(n=5)$ hours after exploration. A control group of animals $(n=5)$ was placed for 20 minutes in the same box in the dark, but without the objects (Fig. 1B), returned to their home cages and then euthanized after $1 \mathrm{~h}$. Home cages were free of objects and the animals were well adapted to them, which precluded that prior environmental stimuli could induce IEG activation. To evaluate the degree of locomotion of the animals during the post-exploratory period, the total distance traveled and the mean velocity were video recorded and quantified using Any-maze software (version 4.3, Stoelting Co., Wood Dale, IL, USA). 
115 Immunohistochemistry: All animals were deeply anaesthetized with sodium pentobarbital

116 (90 mg/kg, i.p.) and decapitated. The brains were quickly removed and fast-frozen in an

117 embedding medium (Tissue Tek, Sakura Finetek, Japan). Then, the brains were frontally

118 sectioned at $20 \mu \mathrm{m}$ in a cryostat (Carl Zeiss Micron HM 550, Germany) and thaw-mounted over

119 electrically charged glass slides following a serial distribution. Brain slices were then post-fixed

120 for $15 \mathrm{~min}$ in $4 \%$ paraformaldehyde in $0.1 \mathrm{M}$ phosphate buffer (PB). For immunohistochemistry,

121 sections were washed during $20 \mathrm{~min}$ in phosphate buffer-Tween (PB-T) and incubated in a

122 blocking buffer $(\mathrm{BB})$ solution $(0.5 \%$ fresh skim milk and $0.3 \%$ Triton $\mathrm{X}-100$ in $0.1 \mathrm{M} \mathrm{PB})$ for 30

123 min. Sections were incubated overnight at $18^{\circ} \mathrm{C}$ in primary antibody (1:200 in BB;

124 phosphorylated polyclonal rabbit CaMKII, Millipore Corp., USA, catalog \#AB3827; rabbit

125 polyclonal egr-1 and c-fos, Santa Cruz Biotechnology, Inc., USA, catalog \#sc-189 and \#sc-52,

126 respectively), washed in PB-T (2x, $10 \mathrm{~min}$ each), incubated with a biotinylated secondary goat

127 anti-rabbit antibody (1:200 in BB, Vector Labs, USA) for 2h, washed in PB-T (2x, 10 min each),

128 and incubated in avidin-biotin-peroxidase solution (Vectastain Standard ABC kit, Vector Labs,

129 USA) for $2 \mathrm{~h}$. Next, slides were placed in a solution containing $0.03 \% 3,3$ ' diaminobenzidine

130 (DAB) and $0.001 \%$ hydrogen peroxide in 0.1M PB (Freire et al., 2005). Primary antibodies were

131 replaced by normal serum in some test sections. The reaction was monitored every 30 min and

132 was interrupted by washing sections in $0.1 \mathrm{M}$ PB during $5 \mathrm{~min}$. In order to avoid artifacts due to

133 procedural inconsistencies, all sections of all groups were processed together, incubated

134 simultaneously and spent an identical time in the same DAB solution. Alternate sections were

135 stained with cresyl violet (Nissl staining) to reveal cyto-architectonic boundaries. At the end of

136 all procedures, sections were dehydrated and cover-slipped with Entellan (Merck, Darmstadt,

137 Germany). Digital images were acquired with a CX9000 camera (MBF Bioscience Inc.,

138 Williston, VT, USA) attached to a Nikon Eclipse 80i optical microscope (Tokyo, Japan - 4x, 10x

139 and 20x objectives) under the same light parameters, to avoid bias. The contrast, and/or

140 brightness of pictures were adjusted using Photoshop CS5 software (Adobe Systems Inc., San

141 José, CA, USA).

142

143 Data quantification: IEG-labeled cells (3 sections/animal, $n=5$ animals per group for every

144 marker) were counted with the Neurolucida system (MBF Bioscience Inc., USA) using 10 grids

145 of $100 \times 100 \mu \mathrm{m}$ for each section across V1 cortex. Analysis of pCaMKII staining in V1 cortex 
146 was done by optical densitometry with the ImageJ software (http://rsb.info.nih.gov/ij/) using a $1470.05 \mathrm{~mm}^{2}$ square window ( $n=5$ animals per group, 3 sections per animal; 5 samples per section).

148 The low reactivity in the white matter was used as background reference (signal was averaged 149 over 5 different sites using the same square window). For each animal, the average optical 150 density (OD) was named G, cortical white matter $\mathrm{W}$ and a contrast index $\mathrm{C}$ was calculated 151 according to the equation: $\mathrm{C}=(\mathrm{G}-\mathrm{W}) /(\mathrm{G}+\mathrm{W})$ (Freire et al., 2007). All photographs were taken 152 with the same illumination and microscope (Nikon Eclipse 80i, Tokyo, Japan). Average values 153 for all measurements were normalized to the levels observed in the control group.

155 Statistics: Significant differences across groups were assessed with the non-parametric Kruskal 156 Wallis test, with Dunn post-hoc test $(\alpha=0.05)$, using the Graphpad Prism 5.0 software (GraphPad 157 Software Inc., La Jolla, CA, USA). The numerical values represented in boxplots were presented 158 as median with indication of the 25 th to 75 th percentiles.

\section{Results}

To ensure that the analyses involved only the region of interest associated with V1 cortex, 162 its boundaries were defined by Nissl staining (Fig. 2A). In a qualitative analysis, 163 immunohistochemistry with specific antibodies revealed a robust increase of egr-1 and c-fos 164 labeling in V1 cortex of animals killed 1h after object exploration in darkness, with a subsequent 165 decrease after $3 \mathrm{~h}$ (Fig. 2B). However, the pattern of activation at the $3 \mathrm{~h}$ time period was still 166 higher than the basal levels found in unexposed controls (Fig. 2B). The levels of phosphorylated 167 CaMKII in V1 cortex followed a similar time course (Fig. 2B).

Although we had not quantified their intensity of immunolabeling in c-fos and egr-1reactive cells, the general pattern of reactivity allowed us to identify cell profiles varying from intensely to weakly reactive in all evaluated time points. There was a marked prevalence of intensely reactive cells $1 \mathrm{~h}$ after exploration, a pattern markedly different from that seen at $3 \mathrm{~h}$, which was more balanced (Fig. 2B). The effectiveness of the immunolabeling method for all markers could be demonstrated by the absence of labeling when the primary antibody was replaced by normal serum (test sections). Since the brains were collected fresh and immediately

175 fast-frozen, some blood normally remains in the tissue. This likely explains the differences in 176 background labeling observed across animals in the present study. 
A quantitatively analysis confirmed that the peak of activity in V1 cortex occurred after

$1781 \mathrm{~h}$ of exploration in darkness for all markers (Fig. 3) (Kruskal-Wallis, Dunn post-hoc test,

$179 * \mathrm{p}<0.05)$. After $3 \mathrm{~h}$ of exploration the expression levels for all markers in V1 cortex were lower

180 when compared to those seen at $1 \mathrm{~h}$ of exploration, being similar to control levels (Fig.

181 3).Locomotion parameters (total traveled distance and mean velocity) measured during the post-

182 exploration period revealed a similar behavioral pattern in all groups (Fig. 4A), with the animals

183 spending equivalent amounts of time moving in the home cage (Fig. 4B).

\section{Discussion}

In the present work, we investigated whether the whisker-based exploration of novel objects in the dark is capable of increasing the protein levels of molecular markers of plasticity in the V1 cortex. In a previous study using the same experimental paradigm, we have demonstrated that it increases the mRNA levels of calcium-dependent IEGs in the V1 cortex (Ribeiro et al., 2007). Here we provide evidence that such non-visual exploration also increases the levels of phosphorylated CaMKII, as well as c-fos and egr-1 proteins. This suggests that the cross-modal response in the V1 cortex can lead to long-term plasticity.

In a recent study in rats, Nakajima and coworkers showed that visual deprivation induces a sustained increase of neural activity in the S1 cortex (Nakajima et al., 2016). The up-regulation of neuronal responsivity in the S1 cortex after visual deprivation can be explained by an strengthening of synaptic activity mediated by NMDA and/or AMPA receptors, which induces IEG expression (Kaczmarek \& Chaudhuri, 1997). Our present results showing IEG activation in V1 cortex after non-visual exploration in the dark are supported by a recent report of long-term spatiotemporal changes in $c$-fos and arc levels in this area after bilateral deafness in adult rats (Pernia et al., 2017). According to this study, the disruption of the auditory input induces thalamocortical reorganization with consequent modification in both auditory and visual cortical circuits, which leads to the overexpression of IEGs in V1 cortex and a further re-expression of these markers in the auditory cortex after thalamocortical reordering (Pernia et al., 2017). A similar pattern of IEG activation was also verified after visual deprivation (Takahata \& Kaas, 204 2016).

The antibodies used have been previously validated (Blanco et al., 2015). The crossmodal up-regulation of the protein levels of egr-1 and c-fos detected in the present study is 
208 Leah, 1998; Lonergan et al., 2010), likely in association with the differential expression of 209 NMDA receptors (Chaudhuri, 1997; Kaczmarek \& Chaudhuri, 1997).

210 The present results add to the notion that cross-modality can be induced by the transient

211 deprivation of stimulus from a sensory modality (visual, in this case), i.e. without any damage to

212 the sensory periphery (Pascual-Leone \& Hamilton, 2001; Merabet et al., 2008). However, since

213 we only evaluated factors involved in a rapid response (one or three hours after visual

214 deprivation), more studies are required to comprehensively characterize this phenomenon

215 downstream of the initial genomic response.

216 Human brain imaging studies have shown that the visual cortex is significantly activated

217 when subjects close their eyes and imagine objects (Kosslyn et al., 1995). Such visual mental

218 imagery occurs when "a visual short-term memory (STM) representation is present but the

219 stimulus is not actually being viewed" (Kosslyn \& Thompson, 2003). In rodents, mental imagery

220 can influence subsequent encoding and recognition processes of landmarks (Karimpur \&

221 Hamburger, 2018). In the present study we cannot rule out that our experimental design triggers

222 imagery in the rats. However, since they explored the objects in the dark without any prior visual

223 presentation, remains to be determined whether visual images could be formed for objects never

224 encountered before.

225 In a previous study with the same experimental design, electrophysiological and 226 molecular changes in V1 cortex were interpreted as nonspecific effects of arousal (Ribeiro et al., 227 2007). Assuming this hypothesis as correct, V1 cortex responses during whisked-based 228 exploration of novel objects in the dark would represent only a general alert signal, carrying no 229 specific information about object identity. However, in a subsequent study we analyzed with 230 more depth the electrophysiological responses of individual V1 and S1 neurons of rats submitted 231 to the same paradigm of exploration in the dark (Vasconcelos et al., 2011). This study revealed 232 no significant difference in V1 and S1 neuronal responses to the explored objects, and thus 233 reinforced the proposal that V1 cortex is in fact recruited during the whisker-based exploration of 234 objects in the dark. Further studies evaluating IEG expression in S1 cortex using a control group 235 with anesthetized whiskers shall help draw a more complete picture of the cellular changes in 236 primary cortical areas whisker-based exploration in the dark.

237 In face of the present results, one key question concerning activation of visual areas by 238 tactile stimulation is: why does it occur? Primary sensory areas have been shown to prefer a 
239 given modality but to quickly engage in cross-modal processing depending on task demands, a 240 property named metamodality (Pascual-Leone \& Hamilton, 2001). The functional recruitment of 241 the visual cortex for tactile processing in healthy adults after a short period of visual deprivation 242 is believe to reflect the unmasking of pre-existing connections (Merabet et al., 2008).

243 The visual cortex can be activated by locomotion (Dipoppa et al., 2018). To assess 244 whether this effect could explain the results we evaluated the locomotion of the animals during 245 the post-novelty period. We found that all groups spent similar amounts of time moving within 246 their home cages, which rules out non-specific activation induced by locomotion.

\section{Conclusions}

248 Our results show that even a short period of dark ( 3 hours) is able to engage neurons in 249 the V1 cortex in non-visual processing, leading to the up-regulation of molecular cascades that 250 link sustained membrane depolarization with long-term gene regulatory changes. Further studies 251 are required to a better understanding of the circuit dynamics associated with IEG activation 252 following visual deprivation. 
255

256

257

258

259

260

261

262

263

264

265

266

267

268

269

270

271

272

273

274

275

276

277

278

279

280

281

282

283

284

285

286

287

288

289

290

291

292

293

294

295

296

297

298

299

300

\section{References}

Bading H, Ginty DD, and Greenberg ME. 1993. Regulation of gene expression in hippocampal neurons by distinct calcium signaling pathways. Science 260:181-186.

Blanco W, Pereira CM, Cota, VR, Souza AC, Rennó-Costa C, Santos S, Dias G, Guerreiro AMG, Tort ABL, Neto, AD, Ribeiro S. 2015. Synaptic homeostasis and restructuring across the sleep-wake cycle. PLoS Comp Biol 11(5):e1004241.

Brodmann K. 2006. Brodmann's localisation in the cerebral cortex the principles of comparative localisation in the cerebral cortex based on cytoarchitectonics. (Vergleichende Lokalisationslehre der Grosshirnrinde in ihren Prinzipien dargestellt auf Grund des Zellenbaues. Leipzig: Johann Ambrosius Barth Verlag; 1909) (L. Garey Trans). New York: Springer.

Chaudhuri A. 1997. Neural activity mapping with inducible transcription factors. Neuroreport 8:iii-vii.

Dipoppa M, Ranson A, Krumin M, Pachitariu M, Carandini M, Harris KD. 2018. Vision and locomotion shape the interactions between neuron types in mouse visual cortex. Neuron 98:602-615.e8

Dooley JC, Franca JG, Seelke AM, Cooke DF, and Krubitzer LA. 2015. Evolution of mammalian sensorimotor cortex: thalamic projections to parietal cortical areas in Monodelphis domestica. Front Neuroanat 8:163.

Freire MAM, Franca JG, Picanço-Diniz CW, and Pereira A, Jr. 2005. Neuropil reactivity, distribution and morphology of NADPH diaphorase type I neurons in the barrel cortex of the adult mouse. $J$ Chem Neuroanat 30:71-81.

Freire MAM, Oliveira RB, Picanco-Diniz CW, and Pereira A. 2007. Differential effects of methylmercury intoxication in the rat's barrel field as evidenced by NADPH diaphorase histochemistry. Neurotoxicology, 28:175-181.

Frostig RD, Xiong Y, Chen-Bee CH, Kvasnak E, and Stehberg J. 2008. Large-scale organization of rat sensorimotor cortex based on a motif of large activation spreads. J Neurosci 28:1327413284.

Guimaraes JS, Santos JR, and Freire MAM. 2016. Korbinian Brodmann: cortical maps and the cytoarchitecture of the brain. Acad $J$ Sur 7:617-620.

Herdegen T, Kovary K, Leah J, and Bravo R. 1991. Specific temporal and spatial distribution of JUN, FOS, and KROX-24 proteins in spinal neurons following noxious transsynaptic stimulation. J Comp Neurol 313:178-191.

Herdegen T, and Leah JD. 1998. Inducible and constitutive transcription factors in the mammalian nervous system: control of gene expression by Jun, Fos and Krox, and CREB/ATF proteins. Brain Res Brain Res Rev 28:370-490.

Hubel DH, and Wiesel TN. 1959. Receptive fields of single neurones in the cat's striate cortex. $J$ Physiol 148:574-591.

Jones MW, Errington ML, French PJ, Fine A, Bliss TV, Garel S, Charnay P, Bozon B, Laroche S, and Davis S. 2001. A requirement for the immediate early gene Zif268 in the expression of late LTP and long-term memories. Nat Neurosci 4:289-296.

Kaczmarek L. 2000. Gene expression in learning processes. Acta Neurobiol Exp (Wars) 60:419424.

Kaczmarek L, and Chaudhuri A. 1997. Sensory regulation of immediate-early gene expression in mammalian visual cortex: implications for functional mapping and neural plasticity. Brain Res Rev 23:237-256. 
301 Karimpur H, and Hamburger K. 2018. A rat in the sewer: How mental imagery interacts with

Kosslyn SM, and Thompson WL. 2003. When is early visual cortex activated during visual mental imagery? Psychol Bull 129:723-746.

Kosslyn SM, Thompson WL, Kim IJ, and Alpert NM. 1995. Topographical representations of mental images in primary visual cortex. Nature 378:496-498.

Lemus L, Hernandez A, Luna R, Zainos A, and Romo R. 2010. Do sensory cortices process more than one sensory modality during perceptual judgments? Neuron 67:335-348.

Lonergan ME, Gafford GM, Jarome TJ, and Helmstetter FJ. 2010. Time-dependent expression of Arc and zif268 after acquisition of fear conditioning. Neural Plast 2010:139891.

Merabet LB, Hamilton R, Schlaug G, Swisher JD, Kiriakopoulos ET, Pitskel NB, Kauffman T, and Pascual-Leone A. 2008. Rapid and reversible recruitment of early visual cortex for touch. PLoS One 3:e3046.

Mountcastle VB. 1997. The columnar organization of the neocortex. Brain 120 (Pt 4):701-722.

Nakajima W, Jitsuki S, Sano A, and Takahashi T. 2016. Sustained enhancement of lateral inhibitory circuit maintains cross modal cortical reorganization. PLoS One 11:e0149068.

Nunes ACL, Duarte RB, Sousa TB, Santos JR, Freire MAM, and Costa MS. 2010. Expression of the immediate-early gene egr-1 and Substance $\mathrm{P}$ in the spinal cord following locomotor training in adult rats. Brain Res 1345:125-136.

Pascual-Leone A, and Hamilton R. 2001. The metamodal organization of the brain. Prog Brain Res 134:427-445.

Pereira A Jr, Freire MAM, Bahia CP, Franca JG, Picanço-Diniz CW. 2000. The barrel field of the adult mouse SmI cortex as revealed by NADPH-diaphorase histochemistry. Neuroreport 11:1889-1892

Pernia M, Estevez S, Poveda C, Plaza I, Carro J, Juiz JM, and Merchan MA. 2017. c-Fos and Arc/Arg3.1 expression in auditory and visual cortices after hearing loss: Evidence of sensory crossmodal reorganization in adult rats. J Comp Neurol 525:2677-2689.

Rabinowitch I, and Bai J. 2016. The foundations of cross-modal plasticity. Commun Integr Biol 9:e1158378.

Ribeiro S, Shi X, Engelhard M, Zhou Y, Zhang H, Gervasoni D, Lin SC, Wada K, Lemos NA, and Nicolelis MA. 2007. Novel experience induces persistent sleep-dependent plasticity in the cortex but not in the hippocampus. Front Neurosci 1:43-55.

Rocha EG, Santiago LF, Freire MAM, Gomes-Leal W, Lent R, Houzel JC, Franca JG, Pereira A, Jr., and Picanço-Diniz CW. 2007. Callosal axon arbors in the limb representations of the somatosensory cortex (SI) in the agouti (Dasyprocta primnolopha). J Comp Neurol 500:255266.

Santiago LF, Rocha EG, Freire MAM, Lent R, Houzel JC, Picanço-Diniz CW, Pereira A, Jr., and Franca JG. 2007. The organizational variability of the rodent somatosensory cortex. Rev Neurosci 18:283-294.

Stehberg J, Dang PT, and Frostig RD. 2014. Unimodal primary sensory cortices are directly connected by long-range horizontal projections in the rat sensory cortex. Front Neuroanat 8:93.

Takahata T, and Kaas JH. 2016. c-FOS expression in the visual system of tree shrews after monocular inactivation. J Comp Neurol 525:151-165.

Van Brussel L, Gerits A, and Arckens L. 2011. Evidence for cross-modal plasticity in adult mouse visual cortex following monocular enucleation. Cereb Cortex 21:2133-2146. 
347 Vasconcelos N, Pantoja J, Belchior H, Caixeta FV, Faber J, Freire MAM, Cota VR, Anibal de Macedo E, Laplagne DA, Gomes HM, and Ribeiro S. 2011. Cross-modal responses in the primary visual cortex encode complex objects and correlate with tactile discrimination. Proc Natl Acad Sci USA 108:15408-15413.

Vincis R, and Fontanini A. 2016. Associative learning changes cross-modal representations in the gustatory cortex. Elife 5.

\section{Figure Captions}

Fig. 1. Experimental design. A) Experimental animals were transferred to a box and allowed to freely explore four novel objects of different shapes and textures ('ball', 'urchin', 'brush' and 'food') in the dark during $20 \mathrm{~min}$, being returned to their regular cages after the end of exploration. B) Control animals were placed for 20 minutes in the same box, but without the objects.

Fig.2. Transient activation of plasticity-related factors in V1 cortex after non-visual object exploration. A) Drawing indicating the location and border V1/V2 and the general aspect of the brain, immediately prior to sectioning. B) Nissl-stained frontal section showing V1 cortex, obtained from the region indicated with the black square in A. The dashed line indicates the border between V1 and V2. C-K) Sections immunostained for egr-1, c-fos and pCaMKII. Controls were unexposed to the objects, and experimental animals were assessed $1 \mathrm{~h}$ and $3 \mathrm{~h}$ after new object exposure. Notice the increased labeling after $1 \mathrm{~h}$ of exposure for all markers. Black squares on low power pictures indicate location from where the enlarged pictures were obtained. Scale bars: $3 \mathrm{~mm}(\mathrm{~A}) ; 200 \mu \mathrm{m}(\mathrm{B}) ; 100 \mu \mathrm{m}(\mathrm{C}-\mathrm{K})$.

Fig.3. Normalized levels plasticity-related factors in V1 cortex after non-visual object exploration. Group data for egr-1, c-fos and pCaMKII, normalized in each panel by the mean value across groups. There was a significant increase $1 \mathrm{~h}$ after object exploration in V1 cortex for all markers ( $n=5$ animals per group, median \pm quartiles of values for all brain sections assessed, Kruskal-Wallis, Dunn post hoc test, ${ }^{*} \mathrm{p}<0.05$ corrected for the number of comparisons).

Fig.4. Locomotion parameters during the post-novelty period. A-B) Quantification of total distance traveled and mean velocity showed that animals of all groups spent similar amount of time moving within their home cages. Blue dot indicates start point, red dot indicates end point. 
380 C) Representative examples of trajectories covered during the post-novelty period. Kruskal381 Wallis, Dunn post hoc test, * $\mathrm{p}>0.05$ corrected for the number of comparisons.

382

383

384 
Figure 1 (on next page)

Experimental design

Experimental animals were transferred to a box and allowed to freely explore four novel objects of different shapes and textures ('ball', 'urchin', 'brush' and 'food') in the dark during $20 \mathrm{~min}$, being returned to their regular cages after the end of exploration. B) Control animals were placed for 20 minutes in the same box, but without the objects. Drawing adapted from (Ribeiro et al., 2007). 


\section{Figure 2 (on next page)}

Transient activation of plasticity-related factors in V1 cortex after non-visual object exploration

A) Drawing indicating the location and border V1/V2 and the general aspect of the brain, immediately prior to sectioning. B) Nissl-stained frontal section showing V1 cortex, obtained from the region indicated with the black square in $A$. The dashed line indicates the border between V1 and V2. C-K) Sections immunostained for egr-1, c-fos and pCaMKII. Controls were unexposed to the objects, and experimental animals were assessed $1 \mathrm{~h}$ and $3 \mathrm{~h}$ after new object exposure. Notice the increased labeling after $1 \mathrm{~h}$ of exposure for all markers. Black squares on low power pictures indicate location from where the enlarged pictures were obtained. Scale bars: $3 \mathrm{~mm}$ (A); $200 \mu \mathrm{m}$ (B); $100 \mu \mathrm{m}$ (C-K). 

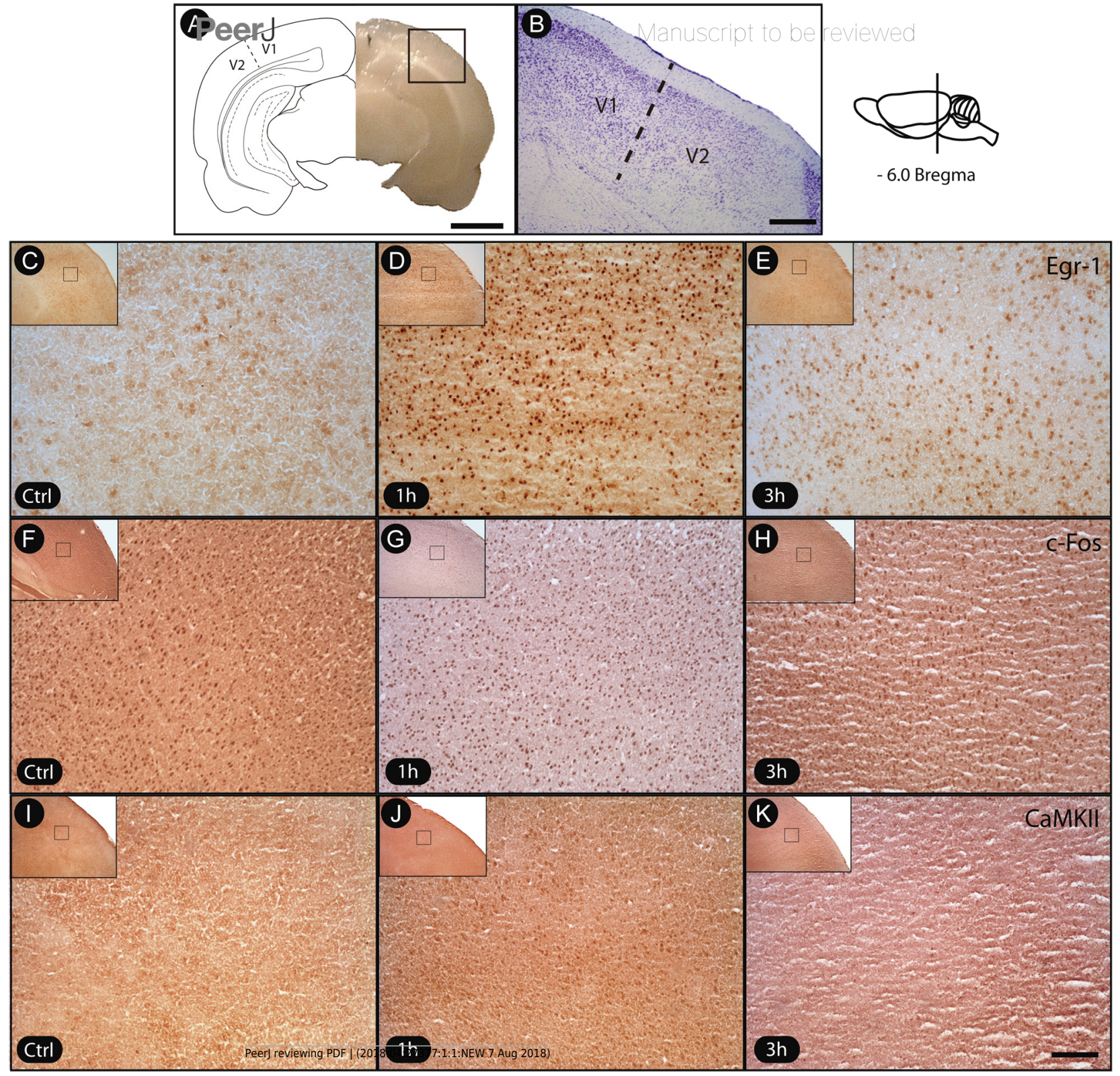
Figure 3 (on next page)

Normalized levels plasticity-related factors in V1 cortex after non-visual object exploration

Group data for egr-1, c-fos and pCaMKII, normalized in each panel by the mean value across groups. There was a significant increase $1 \mathrm{~h}$ after object exploration in V1 cortex for all markers ( $n=5$ animals per group, median \pm quartiles of values for all brain sections assessed, Kruskal-Wallis, Dunn post hoc test, $* p<0.05$ and ${ }^{*} p<0.01$ corrected for the number of comparisons). 
Figure 4 (on next page)

Locomotion parameters during the post-novelty period
A) Quantification of total distance traveled and mean velocity showed that animals of all groups spent similar amount of time moving within their home cages. Blue dot indicates start point, red dot indicates end point. B) Representative examples of trajectories covered during the post-novelty period. Kruskal-Wallis, Dunn post hoc test, $* p>0.05$ corrected for the number of comparisons. 
